\title{
Test-particle dynamics in general spherically symmetric black hole spacetimes
}

\author{
Mariafelicia De Laurentis, ${ }^{1,2,3,4,{ }^{*}}$ Ziri Younsi, ${ }^{1,5}$ Oliver Porth, ${ }^{1}$ Yosuke Mizuno, ${ }^{1}$ and Luciano Rezzolla ${ }^{1,6}$ \\ ${ }^{1}$ Institut für Theoretische Physik, Max-von-Laue-Straße 1, D-60438 Frankfurt am Main, Germany \\ ${ }^{2}$ Universitá di Napoli "Federico II", Compl. Univ. di Monte S. Angelo, \\ Edificio G, Via Cinthia, I-80126 Napoli, Italy \\ ${ }^{3}$ Laboratory of Theoretical Cosmology, Tomsk State University of Control Systems and Radioelectronics \\ (TUSUR), 634050 Tomsk, Russia \\ ${ }^{4}$ INFN Sezione di Napoli, Compl. Univ. di Monte S. Angelo, Edificio G, Via Cinthia, I-80126 Napoli, Italy \\ ${ }^{5}$ Mullard Space Science Laboratory, University College London, \\ Holmbury St. Mary, Dorking, Surrey RH5 6NT, United Kingdom \\ ${ }^{6}$ Frankfurt Institute for Advanced Studies, Ruth-Moufang-Strasse 1, 60438 Frankfurt, Germany
}

(Received 30 November 2017; published 18 May 2018)

\begin{abstract}
To date, the most precise tests of general relativity have been achieved through pulsar timing, albeit in the weak-field regime. Since pulsars are some of the most precise and stable "clocks" in the Universe, present observational efforts are focused on detecting pulsars in the vicinity of supermassive black holes (most notably in the Galactic Centre), enabling pulsar timing to be used as an extremely precise probe of strong-field gravity. In this paper, a mathematical framework to describe test-particle dynamics in general black-hole spacetimes is presented and subsequently used to study a binary system comprising a pulsar orbiting a black hole. In particular, taking into account the parameterization of a general spherically symmetric black-hole metric, general analytic expressions for both the advance of the periastron and for the orbital period of a massive test particle are derived. Furthermore, these expressions are applied to four representative cases of solutions arising in both general relativity and in alternative theories of gravity. Finally, this framework is applied to the Galactic center $S$-stars and four distinct pulsar toy models. It is shown that by adopting a fully general-relativistic description of test-particle motion which is independent of any particular theory of gravity, observations of pulsars can help impose better constraints on alternative theories of gravity than is presently possible.
\end{abstract}

DOI: $10.1103 /$ PhysRevD.97.104024

\section{INTRODUCTION}

It is now widely believed that supermassive black holes (SMBHs) reside at the centers of all galaxies and that their estimated masses are in the range of a few million to up to tens of billions of solar masses. Earth's closest SMBH candidate is found at the Galactic center, Sagittarius A* (Sgr $\mathrm{A}^{*}$ ), which astronomers have been observing for several decades $[1,2]$.

It is expected that the mathematical description of astrophysical black holes (BHs) is based on solutions to the Einstein field equations and therefore founded on general relativity (GR). However, there also exist many $\mathrm{BH}$ solutions in extended and alternative theories of gravity, and to date, observational constraints, most notably in the strong-field regime, are lacking. Moreover, modifications or extensions of classical GR, let alone entirely new theories of gravity, are not without astrophysical

*laurentis@th.physik.uni-frankfurt.de motivation (e.g., accounting for inflation, dark matter, and dark energy) (see [3] for a review).

One promising probe of strong-field gravity is the direct imaging of the shadow cast by a SMBH. High-resolution imaging of the event horizon and $\mathrm{BH}$ shadow can improve understanding of gravity in the strong-field regime and, hopefully, provide direct evidence as to whether BHs exist and which theory (or classes of theory) of gravity describe them best [4-8]. Direct observation of the event horizon of the SMBH Sgr A* will soon be obtained by the Event Horizon Telescope Collaboration (EHTC). ${ }^{1}$ [9-12]. This imaging is performed by combining several radio telescopes into a synchronised, global, and near Earth-sized network using very-long-baseline interferometry (VLBI). However, another important goal of the EHTC is the search for new radio pulsars in the vicinity of Sgr A*. Pulsars provide an additional independent observational tool to help improve the understanding of the properties of Sgr A*

\footnotetext{
${ }^{1}$ See www.eventhorizontelescope.org.
} 
(e.g., its mass, spin, and even geometry), providing considerably stronger constraints than is possible with event horizon-scale imaging alone.

It is well known that pulsars, i.e., rapidly rotating neutron stars, are among the most precise and stable "clocks" in the Universe, providing regular radio signals that can be used to test GR and, in principle, any alternative theory of gravity (see [13] for a comprehensive discussion). In particular, when present in a binary system containing another neutron star or a white dwarf, these objects arguably represent the most promising avenue through which to investigate and constrain large classes of gravity theories [14-16].

The case of a pulsar orbiting around a SMBH is particularly interesting since one can in principle combine precision timing measurements with measurements of geodesic motion around the $\mathrm{BH}$, i.e., in the strong-field regime. This system configuration has proven thus far to be elusive, and consequently intensive searches by observational surveys like BlackHoleCam ${ }^{2}$ and EHTC will prove extremely important in view of these detections. Such timing measurements can contribute to fixing strict ranges on the parameters of a given class of gravity theories and therefore facilitate the selection of viable theories without imposing any a priori assumptions.

Several different gravity theories can explain the same experimental data with almost the same accuracy $[17,18]$. The case of dark matter is paradigmatic: astrophysical effects related to the older concept of "missing matter" by Zwicky [19] can be addressed quite well by modifying the matter sector as well as the gravity sector inside the field equations (see [20] for a comprehensive review). This degeneracy could be removed in favour of one of the two approaches by either discovering new particles or by selecting some "new" gravitational effect that clearly identifies a modified theory. At the same time, it is most desirable that the new gravitational effect is measured in a way that does not rely, a priori, on the selection of a given theory (or class of theories) of gravity.

In order to address this specific problem, several authors have presented novel and general approaches which enable the BH spacetime to be parametrized based on specific perturbations of, or deviations from, the general-relativistic Kerr metric [21-24]. However, in this study theories of gravity entirely distinct from GR are investigated, and consequently, the parametrization of Rezzolla and Zhidenko (RZ) [25] is employed.

The RZ parametrization is a general representation of $\mathrm{BH}$ spacetimes in arbitrary metric theories of gravity. In the case of spherically symmetric spacetimes, the parametrization makes use of a coordinate compactification in terms of a rapidly convergent continued-fraction expansion defined in the radial direction between the event horizon and spatial infinity. A similar approach has also been employed to

\footnotetext{
${ }^{2}$ See blackholecam.org.
}

describe axisymmetric solutions, where the radial expansion is accompanied by an expansion in the polar direction and away from the equatorial plane [26]. In this way, it is possible to represent a given $\mathrm{BH}$ solution to very high accuracy with a small number of free parameters (see $[8,27]$ for some examples of the application of this parametrization to describe $\mathrm{BH}$ shadows).

The focus of the present study is restricted to the spherically symmetric case, and general expressions for the dynamics of a test-particle in general BH spacetimes, such as the motion of a pulsar orbiting around the SMBH candidate Sgr A*, are derived. In particular, explicit general expressions for the advance of the periastron and the orbital period at different orders of the parametrization are provided. Furthermore, periastron-advance formulae are also given for four representative theories, namely, the Reissner-Nordström solution in GR, and alternative theory of gravity solutions from Einstein-Maxwell-Dilaton-Axion, Brans-Dicke, and $f(R)$ theories.

The paper is organized as follows. In Sec. II, the RZ parametrization [25] is briefly reviewed, while Sec. III describes and compares the parametrization of other representative spherically symmetric BH solutions. Section IV discusses the basic properties of test-particle motion around a spherically symmetric $\mathrm{BH}$, outlining the derivation of the expressions for the advance of the periastron, which are then presented in Sec. V. These expressions are then employed in Sec. VI to numerically investigate the values of the expansion parameters in the case of four $S$-stars and four particular representative pulsar toy models around Sgr. A*. Sec. VII is devoted to the discussion and conclusions.

\section{PARAMETRIZATION FRAMEWORK}

In what follows, the RZ parametrization [25] for a general spherically symmetric BH spacetime is briefly reviewed and subsequently used to determine the dynamics of a test particle, ${ }^{3}$ such as a pulsar orbiting around Sgr A*. Unless otherwise stated, geometrized units with $G=c=1$ are used, where $G$ and $c$ are Newton's constant and the speed of light, respectively.

Consider a spherically-symmetric spacetime with the line element given by [25], i.e.,

$$
\begin{aligned}
d s^{2} & =g_{\alpha \beta} d x^{\alpha} d x^{\beta} \\
& =-N^{2}(r) d t^{2}+\frac{B^{2}(r)}{N^{2}(r)} d r^{2}+r^{2} d \Omega^{2},
\end{aligned}
$$

where the signature $(-,+,+,+)$ is adopted. In the following, the metric coefficients will be written differently.

\footnotetext{
${ }^{3}$ Note that the RZ parametrization [25] does not provide any information on the field equations and hence can only be used to describe the motion of a test particle, be it massive (e.g., a pulsar around a SMBH) or massless (e.g., photons constituting the shadow of a $\mathrm{BH}[8])$.
} 
Hereafter, the metric functions $N^{2}(r)$ and $B^{2}(r)$ are recast as $\mathcal{N}(r)$ and $\mathcal{B}(r)$ for ease of computation.

The geodesic equations of motion are derived from the Lagrangian, which may be written as

$$
\begin{aligned}
2 \mathcal{L} & =g_{\alpha \beta} \frac{d x^{\alpha}}{d \tau} \frac{d x^{\beta}}{d \tau} \\
& =-\mathcal{N}(r) \dot{t}^{2}+\frac{\mathcal{B}(r)}{\mathcal{N}(r)} \dot{r}^{2}+r^{2} \dot{\theta}^{2}+r^{2} \sin ^{2} \theta \dot{\varphi}^{2},
\end{aligned}
$$

where $\dot{x}^{\mu}:=d x^{\mu} / d \tau$ is the particle's four-velocity, $\tau$ is the affine parameter along the geodesic (in this work, the proper time), $t$ is the coordinate time, and an overdot denotes differentiation with respect to $\tau$. In the RZ parametrization, the function $\mathcal{N}(r)$ is then expressed as

$$
\mathcal{N}(x)=x A(x),
$$

where

$$
A(x)>0 \text { for } 0 \leq x \leq 1,
$$

with

$$
x:=1-\frac{r_{0}}{r},
$$

so that $x=0$ is the position of the event horizon and $x=1$ corresponds to spatial infinity. Furthermore, $A$ and $B$ may be expressed in terms of the parameters $\epsilon, a_{i}$, and $b_{i}$ ( $i \in[0, n]$, where $n$ is the expansion order), such that

$$
\begin{gathered}
A(x)=1-\epsilon(1-x)+\left(a_{0}-\epsilon\right)(1-x)^{2}+\tilde{A}(x)(1-x)^{3}, \\
\mathcal{B}(x)=1+b_{0}(1-x)+\tilde{B}(x)(1-x)^{2},
\end{gathered}
$$

where the functions $\tilde{A}$ and $\tilde{B}$ describe the metric near the horizon (i.e., $x \simeq 0$ ) and at spatial infinity (i.e., $x \simeq 1$ ). It is evident that the metric is finite in both limits [25].

The functions (6a)-(6b) can then be expanded via a Padé approximation of continued fractions as

$$
\begin{aligned}
& \tilde{A}(x)=\frac{a_{1}}{1+\frac{a_{2} x}{1+\frac{a_{3} x}{1+\cdots}}}, \\
& \tilde{B}(x)=\frac{b_{1}}{1+\frac{b_{2} x}{1+\frac{b_{3} x}{1+\cdots}}},
\end{aligned}
$$

where $a_{1}, a_{2}, \ldots, a_{n}$ and $b_{1}, b_{2}, \ldots, b_{n}$ are dimensionless constants that can be fixed once the generalised metric (1) is matched to a specific metric. Hereafter, to keep expressions compact, all calculations will be performed up to third order in the above expansion; already at this order the differences between the matched metric and the exact metric are below $1 \%[8,25,28]$. Finally, the parameter $\epsilon$ in equations (6a)-(6b) measures the deviations of the position of the event horizon in the general metric from the corresponding location in a Schwarzschild spacetime, i.e.,

$$
\epsilon=\frac{2 M-r_{0}}{r_{0}}=-\left(1-\frac{2 M}{r_{0}}\right) .
$$

\section{APPLICATIONS OF THE RZ PARAMETRIZATION}

There are several BH solutions which differ from GR, and in order to perform a fair and unbiased analysis of the framework presented in this study, in what follows no one model is favoured above others, even though in practice there are physical motivations to do so depending on the astrophysical applications in mind. Failure to do so would require repeating the analysis for any and all models, which would be time consuming and impractical. In the following, the advantage of this parameterized approach which enables one to mimic different $\mathrm{BHs}$ is demonstrated. In particular, four different representative $\mathrm{BH}$ solutions are chosen to illustrate this.

First, a well-known spherically symmetric solution of GR is considered, namely, the Reissner-Nordström BH, which is in itself interesting because it contains an electric charge. The presence of this electric charge implies that the Reissner-Nordström $\mathrm{BH}$ can be more compact than a Schwarzschild BH of the same mass. Second, scalar-tensor theories, such as Brans-Dicke theory and $f(R)$ theories, which are a major focus of a large proportion of the gravitational physics community since they represent simple deviations from (or extensions of) GR are next considered, providing interesting examples of modified gravity theories. Finally, a solution containing a dilaton scalar field, i.e., an Einstein-Maxwell-Axion-Dilaton BH, is also investigated.

\section{A. Einsteinian gravity: Reissner-Nordström}

The Reissner-Nordström metric describes the geometry of a spherically symmetric and charged $\mathrm{BH}$ [29]. The line element can then be written in the form (1) with

$$
\mathcal{N}(r)=1-\frac{2 M}{r}+\frac{r_{\mathrm{Q}}^{2}}{r^{2}}, \quad \mathcal{B}(r)=1,
$$

and $r_{\mathrm{Q}}$ is a characteristic length scale given by

$$
r_{\mathrm{Q}}^{2}=\frac{G Q^{2}}{4 \pi \varepsilon_{0}} c^{4},
$$

where $\left(4 \pi \varepsilon_{0}\right)^{-1}$ is the Coulomb force constant. In the limit $Q \rightarrow 0$, one recovers the Schwarzschild solution. 
The Reissner-Nordström solution possesses two event horizons, which are located at

$$
r_{0_{\mathrm{RN}}}=M \pm \sqrt{M^{2}-r_{Q}^{2}}
$$

Combining the above relation with (8), one may write $\epsilon$ for the Reissner-Nordström solution as

$$
\epsilon_{\mathrm{RN}}=\frac{2 M}{M+\sqrt{M^{2}-r_{\mathrm{Q}}^{2}}}-1 .
$$

Upon expanding the metric coefficients (9) at spatial infinity, comparison with the RZ parametrization yields at zeroth order

$$
a_{0, \mathrm{RN}}=\frac{r_{\mathrm{Q}}^{2}}{\left[\sqrt{M^{2}-r_{\mathrm{Q}}^{2}}+M\right]^{2}}, \quad b_{0_{\mathrm{RN}}}=0 .
$$

Similarly, comparing the behaviour of the coefficients (9) near the horizon, for all expansion orders $i \geq 1$, the following may be deduced

$$
a_{i, \mathrm{RN}}=0, \quad b_{i, \mathrm{RN}}=0 .
$$

In other words, the Reissner-Nordström metric is fully represented by the RZ parametrization at the zeroth order of the expansion.

\section{B. Alternative theories of gravity}

Alternative theories of gravity consider, in general, further (minimally or nonminimally coupled) scalar fields or higher-order curvature or torsion invariants in the Hilbert-Einstein Lagrangian. For example, if correction terms such as $\phi^{2} R, R^{2}, R_{\alpha \beta} R^{\alpha \beta}, R_{\alpha \beta \gamma \delta} R^{\alpha \beta \gamma \delta}$, and $R \square R$ are incorporated in the Lagrangian, they give rise to modified gravitational dynamics [3,30-33].

In particular, these modifications have been introduced in order to alleviate problems at ultraviolet scales (e.g., divergences in quantum field theory, the lack of a selfconsistent quantum gravity theory, etc.) and at infrared scales (e.g., the cosmological accelerated expansion dubbed the "dark energy problem" and the clustering properties of large-scale structure, dubbed the "dark-matter problem" [20].

In particular, it is desirable to be able to calculate astrophysically observable quantities in a way that does not rely on making any assumption as to a particular theory of gravity. In this context, the RZ parametrization provides a general approach that is independent of the assumptions pertaining to a given theory of gravity and describes the properties of test-particle motion, e.g., the advance of the periastron, simply in terms of the coefficients $a_{i}$ and $b_{i}$. To this end and principally in order to provide working examples, in the following subsections three different classes of BH solution in alternative theories of gravity are considered, namely, Brans-Dicke theory, $f(R)$ gravity, and Einstein-Maxwell-Axion-Dilaton gravity.

\section{Brans-Dicke theory}

Brans-Dicke (BD) theory is the most well-known of the scalar-tensor theories. In BD theory, the gravitational interaction is mediated by a scalar field. The gravitational coupling is no longer constant, and instead, $1 / G$ is replaced by a scalar field $\phi$ which is nonminimally coupled to the Ricci scalar $R$ [34]. The BD field equations contain a kinetic parameter, $\omega$, termed the BD coupling constant. This is a dimensionless constant related to the strength and variability of the scalar field, and whose value can be chosen to fit observations. Using Solar-System tests, it is possible to impose lower and upper bounds on the possible values for $\omega$. Stringent limits on $\omega$ can also be achieved through a consideration of the dynamics of binary pulsars, and some BH solutions can be derived in the framework of this theory [35-39]. Hereafter, the following BD solution is considered $^{4}$ :

$d s^{2}=-A(r)^{m+1} d t^{2}+A(r)^{n-1} d r^{2}+r^{2} A(r)^{n} d \Omega^{2}$,

with $A(r)=1-2 \tilde{r} / r$, where $\tilde{r}, m$ and $n$ are arbitrary constants. The scalar field is given by

$$
\phi(r)=\phi_{0} A(r)^{-(m+n) / 2},
$$

with $\phi_{0}$ a constant. It is important to emphasise that the parameter $n$ has the role of scalar hair and that as soon as $n=0$ the no-hair condition is restored. It is immediately clear that for $m=n=0$ the Schwarzschild solution is recovered, with asymptotic flatness being recovered for any value of $m$ and $n$. The BD parameter is found from the following relation:

$$
\omega=-2\left(1+\frac{m-n-m n}{(m+n)^{2}}\right) .
$$

In this manner, for each assigned value of $m$ and $n$, a class of $\mathrm{BH}$ solutions is obtained. The event horizon is given by

$$
r_{0, \mathrm{BD}}=2 \tilde{r} \quad \forall m-n+1>0 .
$$

The parameter $\tilde{r}$ may be identified with $k M$, where $M$ is the $\mathrm{BH}$ mass, and $k$ is an arbitrary constant, yielding different

\footnotetext{
${ }^{4}$ Note that these solutions are usually reported to be that of black holes; however, according to the values of the parameter $n$ they can be shown to describe wormholes (i.e., $n>-1$ ) or naked singularities [40]. Since we consider here the case of $n=0$ (for which the throat of the wormhole acts as an apparent horizon) and are interested only in the exterior spacetime, this represents a perfectly useful solution.
} 
event horizons. For example, if $k=1,(18)$ corresponds to the Schwarzschild event horizon and therefore $\epsilon=0$, as in the case of GR. Fixing $n=0$, equation (15) reduces to

$$
d s^{2}=-A(r)^{m+1} d t^{2}+A(r)^{-1} d r^{2}+r^{2} d \Omega^{2},
$$

and it is this particular case that is hereafter considered. As a result, $\epsilon$ in this particular BD metric can be expressed as

$$
\epsilon_{\mathrm{BD}}=-\left(1-\frac{M}{\tilde{r}}\right)
$$

In general, expanding $A(r)$ in (15) at infinity yields the following expressions for $a_{0}$ and $b_{0}$ in terms of BD theory as

$$
a_{0, \mathrm{BD}}=\frac{\omega+3(\omega+2)^{2} \epsilon_{\mathrm{BD}}}{2(\omega+2)^{2}}, \quad b_{0, \mathrm{BD}}=0 .
$$

Furthermore, expanding near the horizon yields the following relations for the parameters in terms of the theory are obtained

$$
a_{1, \mathrm{BD}}=\frac{(\omega-2) \omega+3(\omega+2)^{3} \epsilon_{\mathrm{BD}}}{6(\omega+2)^{3}},
$$

with

$$
a_{2, \mathrm{BD}}=0, \quad \text { and } \quad b_{1, \mathrm{BD}}=b_{2, \mathrm{BD}}=0 .
$$

Therefore, BD theory is represented by the RZ parametrization at the first order of the expansion.

\section{2. $f(R)$ gravity}

The general class of $f(R)$ theories relax the hypothesis that the Hilbert-Einstein action must be linear in the Ricci scalar and instead assume general functions of $R$ that may be constrained by observations and through theoretical considerations [41]. Such theories can always be reduced to scalar-tensor theories by conformal transformations [3]. Due to this property, the above scheme can also be adopted here, and in particular, the parametrized post-Newtonian $(\mathrm{PPN})^{5}$ parametrization arising from $f(R)$ gravity can be straightforwardly related to the RZ parametrization. Assuming a static and spherically symmetric metric, a general post-Newtonian (PN) approximation can be written as [43]

\footnotetext{
${ }^{5}$ The post-Newtonian (PN) approach is an analytical approximation to GR based on a power series expansion in terms of the ratio $v / c$, where $v$ is the typical velocity of the system. In the limit where $v$ becomes infinite, the PN expansion reduces to Newton's law of gravity. The PPN parametrization uses the PN expansion to explicitly detail the parameters in which a general theory of gravity can differ from Newtonian gravity [42].
}

$$
d s^{2}=-\mathcal{N}(r) d t^{2}+\left(1+\gamma \frac{2 M}{r}\right) d r^{2}+r^{2} d \Omega^{2},
$$

where

$$
\mathcal{N}(r)=1-\frac{2 M}{r}+\frac{\beta-\gamma}{2}\left(\frac{2 M}{r}\right)^{2}
$$

The parameters $\gamma$ and $\beta$ provide a measure of the degree of curvature of spacetime as generated by a body of mass $M$ at radius $r$. Equation (24) is general and is valid for any metric theory within which it is possible to derive the PN limit. This means that metric coefficients and subsequently PN parameters strictly depend on the choice of theory. The RZ parametrization can serve as a useful approach in selecting viable $f(R)$ models within the PN-approximation. Generalized PN-parameters can then be expressed in terms of $f(R)$ theory as [44-46]

$$
\begin{gathered}
\gamma_{\mathrm{f}(\mathrm{R})}=1-\frac{\left[f^{\prime \prime}(R)\right]^{2}}{f^{\prime}(R)+2\left[f^{\prime \prime}(R)\right]^{2}}, \\
\beta_{\mathrm{f}(\mathrm{R})}=1-\frac{1}{4}\left(\frac{f^{\prime}(R) f^{\prime \prime}(R)}{2 f^{\prime}(R)+3\left[f^{\prime \prime}(R)\right]^{2}}\right) \frac{d \gamma_{\mathrm{f}(\mathrm{R})}}{d R} .
\end{gathered}
$$

It is evident that $\gamma_{\mathrm{f}(\mathrm{R})}$ and $\beta_{\mathrm{f}(\mathrm{R})}$ are strictly dependent on the function $f(R)$ and its derivatives. Here, $f^{\prime}(R):=d f(R) / d R$. It is straightforward to demonstrate that for $R \rightarrow \phi$, using a conformal transformation, one recovers immediately the results for scalar tensor (ST) theories obtained in [47]:

$$
\begin{gathered}
\gamma_{\mathrm{ST}}=1-\frac{\left[F^{\prime}(\phi)\right]^{2}}{F(\phi)+2\left[F^{\prime}(\phi)\right]^{2}}, \\
\beta_{\mathrm{ST}}=1-\frac{1}{4}\left(\frac{F(\phi) F^{\prime}(\phi)}{2 F(\phi)+3\left[F^{\prime}(\phi)\right]^{2}}\right) \frac{d \gamma_{\mathrm{ST}}}{d \phi} .
\end{gathered}
$$

Here, $\gamma_{\mathrm{ST}}$ and $\beta_{\mathrm{ST}}$ depend on the non-minimal coupling function $F(\phi)$ and its derivatives, and the parameter $\alpha$ determines the deviation with respect to GR. As a general consideration, it is possible to say that both Eqs. (26) and (27) or Eqs. (28) and (29) parameterize modified theories of gravity according to a higher-order approach (e.g., $f(R)$ ) or a ST approach. The key point is that both pictures can be recast in terms of the RZ parametrization and thus expressed in a general approach which is effectively independent of the theory.

Comparing the expansions of the metrics (1) and (24) at the same order, it may be deduced that the event horizon for a general $f(R)$ model is given by

$$
r_{0, \mathrm{f}(\mathrm{R})}=M+M \sqrt{2 \gamma_{\mathrm{f}(\mathrm{R})}-2 \beta_{\mathrm{f}(\mathrm{R})}+1},
$$

where it is straightforward to see that when $f(R)=R$, the Schwarzschild event horizon is readily recovered. Similarly, the expression for $\epsilon$ in a $f(R)$ theory is found as 


$$
\epsilon_{\mathrm{f}(\mathrm{R})}=-\left(1-\frac{2 M}{r_{0, \mathrm{f}(\mathrm{R})}}\right) .
$$

Finally, asymptotically expanding the metrics (1) and (24) and collecting the terms at equivalent expansion orders yields the lowest-order expansion coefficients $a_{i, \mathrm{f}(\mathrm{R})}$ and $b_{i, \mathrm{f}(\mathrm{R})}$ as

$$
\begin{gathered}
a_{0, \mathrm{f}(\mathrm{R})}=\frac{\left(\beta_{\mathrm{f}(\mathrm{R})}-\gamma_{\mathrm{f}(\mathrm{R})}\right)\left(1+\epsilon_{\mathrm{f}(\mathrm{R})}\right)^{2}}{2}, \\
b_{0, \mathrm{f}(\mathrm{R})}=\frac{\left(\gamma_{\mathrm{f}(\mathrm{R})}-1\right)\left(1+\epsilon_{\mathrm{f}(\mathrm{R})}\right)}{2}, \\
a_{1, \mathrm{f}(\mathrm{R})}=3\left(a_{0, \mathrm{f}(\mathrm{R})}-\epsilon_{\mathrm{f}(\mathrm{R})}\right), \\
b_{1, \mathrm{f}(\mathrm{R})}=-1-b_{0, \mathrm{f}(\mathrm{R})}+\sqrt{1+\epsilon_{\mathrm{f}(\mathrm{R})}+2 b_{0, \mathrm{f}(\mathrm{R})}}, \\
a_{2, \mathrm{f}(\mathrm{R})}=\frac{1}{a_{1, \mathrm{f}(\mathrm{R})}}\left[3\left(\epsilon_{\mathrm{f}(\mathrm{R})}-1\right)-a_{0, \mathrm{f}(\mathrm{R})}\right], \\
b_{2, \mathrm{f}(\mathrm{R})}=-2+\frac{1}{b_{1, \mathrm{f}(\mathrm{R})}}\left[\frac{\left.1+\epsilon_{\mathrm{f}(\mathrm{R})}+2 b_{0, \mathrm{f}(\mathrm{R})}-b_{0, \mathrm{f}(\mathrm{R})}\right]}{2+b_{0, \mathrm{f}(\mathrm{R})}} .\right.
\end{gathered}
$$

As an example, a straightforward extension of any analytic $f(R)$ theory is the inclusion of a quadratic correction in the Ricci scalar, the simplest correction to the standard EinsteinHilbert action (see [20] for details on other and more complex expressions for $f(R)$ ). In this case, $f(R)$ is simply expressed as a Taylor series truncated at second order, i.e.,

$$
f(R)=R+\alpha R^{2}+\cdots .
$$

Such a class of $f(R)$ theories has several applications, ranging from Solar-System scales [48-50] up to earlyUniverse cosmology [51]. However, it is important to consider that the range of values of $\alpha$ strictly depends on the scales under consideration. For instance, it can be related to the scalaron or inflaton mass, and it must be compatible with the observed amplitude of scalar perturbations, i.e., in agreement with Planck data $[17,18]$. In this work, however, the length scales considered are much smaller than cosmological scales, and so the resulting values for $\alpha$ are not those normally adopted in the literature, e.g., assuming dimensional units, a standard value for $\alpha$ can be $1 / 6$, as obtained from conformal transformations [see [52], for further details]. Nevertheless, theories of this type have been used recently to describe gravitational corrections around SMBHs [53-55].

\section{Einstein-Maxwell-Dilaton-Axion}

The third alternative gravity theory considered in this work is the spherically symmetric form of the EinsteinMaxwell-Dilaton-Axion (EMDA) gravity [56,57]. In particular, the EMDA metric considered in this study is spherically symmetric and is constructed from a simplification of the axisymmetric EMDA solution [58] in the case of a vanishing axion field. Solutions of this type arise from string theory [59-63]. When the axion field vanishes and the BH is spherically symmetric, the EMDA BH is sometimes referred to as a "dilaton" BH, and the line element takes the following form

$d s^{2}=-\left(\frac{r-2 \mu}{r+2 \hat{b}}\right) d t^{2}+\left(\frac{r+2 \hat{b}}{r-2 \mu}\right) d \rho^{2}+\left(r^{2}+2 \hat{b} r\right) d \Omega^{2}$,

where

$$
\mu:=M-\hat{b} .
$$

Here, $\hat{b}$ is the dilaton parameter and $\mathrm{M}$ the $\mathrm{BH}$ mass (see [63]). Upon recasting the radial coordinate as

$$
\rho^{2}=r^{2}+2 \hat{b} r,
$$

the EMDA line element may be re-expressed in terms of the RZ metric as

$$
\mathcal{N}(\rho)=1-\frac{2 M \rho}{r^{2}}, \quad \mathcal{B}(\rho)=\frac{r^{2}}{\hat{b}^{2}+r^{2}},
$$

where $r \equiv r(\rho)$. Recalling that the location of the event horizon for this $\mathrm{BH}$ is given by

$$
\rho_{0, \mathrm{DIL}}=2(M-\hat{b}),
$$

and using Eq. (8), the expression for $\epsilon$ in terms of the axiondilaton parameters may be written as

$$
\epsilon_{\mathrm{DIL}}=\sqrt{1+\frac{\hat{b}}{\mu}}-1,
$$

where the subscript "DIL" refers to the dilaton BH. In a similar manner, expanding the metric coefficients at spatial infinity gives the values for $a_{0}$ and $b_{0}$ as

$$
a_{0, \mathrm{DLL}}=\frac{\hat{b}}{2 \mu}, \quad \text { and } \quad b_{0, \mathrm{DLL}}=0
$$

In order to obtain the remaining coefficients, one must instead compare the near-horizon expansions, obtaining

$$
\begin{gathered}
a_{1, \mathrm{DIL}}=-3-a_{0, \mathrm{DIL}}+2\left(\epsilon_{\mathrm{DIL}}+1\right)+\left(1+a_{0, \mathrm{DLL}}\right)^{-1}, \\
b_{1, \mathrm{DIL}}=\frac{\epsilon_{\mathrm{DIL}}+1}{1+a_{0, \mathrm{DLL}}}-1,
\end{gathered}
$$




$$
\begin{gathered}
a_{2, \mathrm{DIL}}=\frac{2\left(a_{0, \mathrm{DLL}} \epsilon_{\mathrm{DIL}}-a_{0, \mathrm{DIL}}^{2}+\epsilon_{\mathrm{DIL}}\right)+1}{2\left(a_{0, \mathrm{DIL}}+1\right)^{2}}, \\
b_{2, \mathrm{DIL}}=b_{1, \mathrm{DIL}}-\frac{\hat{b}^{2}}{\left(1+a_{0, \mathrm{DIL}}\right)^{2}} .
\end{gathered}
$$

Note that the Schwarzschild BH solution is recovered from the dilaton $\mathrm{BH}$ solution in the limit of vanishing dilaton parameter.

\section{MOTION AROUND A SPHERICALLY SYMMETRIC BLACK HOLE}

With the formalism derived so far, the calculation of particle trajectories in the neighbourhood of a general spherically symmetric BH may now be calculated. The canonical momenta may be expressed in terms of the RZ parametrization as

$$
\begin{gathered}
p_{t}:=\frac{\partial \mathcal{L}}{\partial \dot{t}}=-\mathcal{N}(r) \dot{t}, \\
p_{r}:=\frac{\partial \mathcal{L}}{\partial \dot{r}}=\frac{\mathcal{B}(r)}{\mathcal{N}(r)} \dot{r}, \\
p_{\theta}:=\frac{\partial \mathcal{L}}{\partial \dot{\theta}}=r^{2} \dot{\theta}, \\
p_{\varphi}:=\frac{\partial \mathcal{L}}{\partial \dot{\varphi}}=r^{2} \sin ^{2} \theta \dot{\varphi} .
\end{gathered}
$$

Because of spherical symmetry, any orbital plane may be taken to be the equatorial plane and therefore $\theta=\pi / 2$, and $\dot{\theta}=\ddot{\theta}=0$ is assumed without loss of generality. The integrals (constants) of motion may be written as

$$
\begin{gathered}
p_{t}=\mathcal{N}(r) \frac{d t}{d \tau}=-E, \\
p_{\varphi}=r^{2} \frac{d \varphi}{d \tau}=L,
\end{gathered}
$$

where $L$ denotes the component of the angular momentum of the particle projected along the axis perpendicular to the orbital plane.

Furthermore, for motion in the equatorial plane, the total angular momentum coincides with the azimuthal angular momentum. Using Eqs. (54) and (55), the Lagrangian can be rewritten as

$$
2 \mathcal{L}=-\frac{E^{2}}{\mathcal{N}(r)}+\frac{\mathcal{B}(r)}{\mathcal{N}(r)} \dot{r}^{2}+\frac{L^{2}}{r^{2}}=m^{2} .
$$

where $m^{2}=(-1,0,+1)$ depending on whether the motion is timelike, null or spacelike, respectively. Since the motion of a massive particle around a SMBH is considered, $m^{2}=1$ is assumed hereafter. In this case, the constants of motion take the form

$$
\begin{gathered}
E^{2}=\mathcal{N}(r)\left(\frac{L^{2}}{r^{2}}+1\right)+\mathcal{B}(r) \dot{r}^{2}, \\
L=r^{2} \dot{\varphi} .
\end{gathered}
$$

\section{CONNECTING TO PULSAR OBSERVATIONS}

\section{A. Periastron advance and orbital period}

As a direct application of the framework developed in the previous sections, the transition between two close inner orbital turning points (or, equivalently, between two close outer turning points) is calculated. The orbits in this case remain closed if the magnitude $\Delta \varphi$ of the angle swept out by the orbit is $2 \pi$. If this is not the case, then the inner turning points are precessing, and the amount of this precession per orbit is

$$
\delta \varphi_{\mathrm{prec}}=\Delta \varphi-2 \pi .
$$

In order derive the precession, $r$ must be expressed as a function of $\varphi$ or vice versa. Combining Eqs. (57)-(58) one obtains

$$
\left(\frac{d r}{d \varphi}\right)^{2}=\mathcal{C} r^{2}\left[\left(\frac{E^{2}}{L^{2}}\right) r^{2}-\left(1+\frac{r^{2}}{L^{2}}\right) \mathcal{N}(r)\right],
$$

where

$$
\mathcal{C}:=\frac{1}{\mathcal{B}(r)}
$$

The angle $\Delta \varphi$ can be computed as the angle swept out as the particle passes between the turning points $r_{1}$ and $r_{2}$ during its orbit, i.e.,

$$
\Delta \varphi=\int_{r_{1}}^{r_{2}} d r \sqrt{\left(\frac{d \varphi}{d r}\right)^{2}},
$$

with the turning points $r_{1}$ and $r_{2}$ being determined from where $\dot{r}=0$ along the orbit.

To illustrate how to derive a theory-independent expression for the periastron advance within the RZ parametrization, first consider the expansion of the metric when the only nonzero expansion terms are $a_{0}$ and $b_{0}$. Analyzing Eq. (60) and remembering that the bound (or unbound) nature of the orbits is determined by the energy $E$, it is assumed that $E^{2}<1$, i.e., the class of orbits considered herein are characterised by a negative energy (i.e., bound orbits). Due to the algebraic complexity of the parametrization and the resulting equations of motion, only the 
general expression is reported, and thus Eq. (60) may be written as

$$
\left(\frac{d r}{d \varphi}\right)^{2}={ }^{(0)} \mathcal{C} r^{2}\left[\left(\frac{{ }^{(0)} E^{2}}{L^{2}}\right) r^{2}-\left(1+\frac{r^{2}}{L^{2}}\right){ }^{(0)} \mathcal{N}(r)\right]
$$

where ${ }^{(0)} \mathcal{C}=\left(1+b_{0} r_{0} u\right)^{-2}$ and ${ }^{(n)}$ indicates working with all expansion coefficients up to $a_{n}$ and $b_{n}$, i.e., up to $n$-th order in the RZ parametrization, ${ }^{(0)} E$ is the energy related to the Lagrangian expanded to zeroth order, and $L$ is independent of the expansion order. Upon changing the variable as $u:=1 / r$ and performing the necessary algebraic simplifications, one obtains

$$
\begin{aligned}
\left(\frac{}{(0)}\left(\frac{d u}{d \varphi}\right)^{2}=\right. & { }^{(0)} \mathcal{C}\left[\frac{{ }^{(0)} E^{2}-1}{L^{2}}+\frac{r_{0}}{L^{2}} u-u^{2}+2 r_{0} u^{3}\right. \\
& -\frac{a_{0} r_{0}^{2}}{L^{2}} u^{2}+\frac{\left(a_{0}-\epsilon\right) r_{0}^{3}}{L^{2}} u^{3}-a_{0} r_{0}^{2} u^{4} \\
& \left.-\left(a_{0}-\epsilon\right) r_{0}^{3} u^{5}\right] .
\end{aligned}
$$

which further modifies the expansion for spacetimes with nonzero $\mathcal{B}$. It is important to emphasize that in general all the $b_{n}$ terms disappear and that only the $a_{n}$ terms remain in the computation of the orbits. Assuming $r_{0}=2 M$ and $a_{0}=b_{0}=\epsilon=0$, the well-known expression for the Schwarzschild geodesic equations in terms of the variable $u$ are readily obtained $[64,65]$. Such expressions may be simplified further by considering the following ansatz

$$
u=\frac{1+e \cos \chi}{2 \ell},
$$

where $e$ is the eccentricity and $\ell$ the semilatus rectum of the orbital ellipse. Here, $\chi$ is the so-called eccentric anomaly or relativistic true anomaly [29,66-69]. It is straightforward to express all the elements of orbits as integrals using $\chi$ as the independent variable. According to Eq. (65), at apoastron $\chi=\pi$, and at periastron $\chi=0$. It may be verified that through several substitutions, Eq. (63) reduces to the form

$$
\begin{aligned}
(0)\left(\frac{d \chi}{d \varphi}\right)^{2}= & {\left[\left(\frac{d \chi}{d \varphi}\right)_{\mathrm{GR}}^{2}+(1+e \cos \chi)\left(2 e \mu\left(\epsilon-a_{0}\right) \cos \chi\right.\right.} \\
& \left.-2 a_{0} \mu+2 \mu \epsilon+\epsilon\right)\left(2-2 e^{2} \mu^{2}+8 \mu^{2}-\mu e^{2}\right. \\
& \left.-8 \mu) e^{2} \mu\left(4 e \mu \sin ^{2} \chi \cos \chi+(1-6 \mu) \cos 2 \chi\right)\right] \\
& \times\left[2 b_{0} \mu(1+e \cos \chi)+1\right]^{-2},
\end{aligned}
$$

where $\sigma:=r_{\mathrm{g}} / \ell$, and $r_{\mathrm{g}}$ is the gravitational radius of the $\mathrm{BH}$. Here, the general-relativistic contribution to the periastron advance is given by [29]

$$
\left(\frac{d \chi}{d \varphi}\right)_{\mathrm{GR}}^{2}=1-\sigma(3+e \cos \chi)
$$

which is immediately recovered from (66) when $a_{0}=$ $b_{0}=\epsilon=0$. Upon integrating Eq. (66), considering the semimajor axis $a$ of the orbital ellipse, and using the relation $\ell=a\left(1-e^{2}\right)$, the expression for the periastron advance, $\Delta \varphi$, at zeroth order in the RZ parametrization is obtained as

$$
{ }^{[0]} \Delta \tilde{\varphi}:={ }^{(0)} \Delta \tilde{\varphi}-\Delta \tilde{\varphi}_{\mathrm{GR}},
$$

with

$$
{ }^{[0]} \Delta \tilde{\varphi}=2 \sigma\left(2 a_{0}+4 b_{0} \epsilon+b_{0}+2 \epsilon\right),
$$

and where $\Delta \tilde{\varphi}_{\mathrm{GR}}=3 \sigma, \Delta \tilde{\varphi}:=\Delta \varphi / 2 \pi$.

In order to highlight the contributions at the different orders hereafter, we introduce the following notation: ${ }^{[n+1]} \Delta \tilde{\varphi}:={ }^{(n+1)} \Delta \tilde{\varphi}-{ }^{(n)} \Delta \tilde{\varphi}$, where $\quad{ }^{(n)} \Delta \tilde{\varphi}=\sum_{i=0}^{n}{ }^{[i]} \Delta \tilde{\varphi}$. The first term in the above equation is the purely GR contribution, while the remaining two terms represent the deviations from GR in a general BH spacetime at zeroth order in the RZ parametrization. Following the same procedure, after some simplification the periastron advance may also be obtained for any order of the approximation. For zeroth order, using Eq. (60) and expanding to first order, one obtains upon integration the following expression

$$
{ }^{[1]} \Delta \tilde{\varphi}:={ }^{(1)} \Delta \tilde{\varphi}-{ }^{(0)} \Delta \tilde{\varphi},
$$

with

$$
\begin{aligned}
{ }^{[1]} \Delta \tilde{\varphi}= & 4 \sigma^{2}\left[2\left(a_{1}-3 b_{0}+b_{1}+\epsilon\right)-4 a_{0}\left(b_{0}-1\right)\right. \\
& \left.-3 b_{0}^{2}(1+2 \epsilon)-4 b_{0} \epsilon+4 b_{1} \epsilon\right] .
\end{aligned}
$$

Finally, the periastron advance may be determined up to second order in the expansion. After the necessary work, this is obtained as

$$
{ }^{[2]} \Delta \tilde{\varphi}:={ }^{(2)} \Delta \tilde{\varphi}-{ }^{(1)} \Delta \tilde{\varphi},
$$

with

$$
\begin{aligned}
{ }^{[2]} \Delta \tilde{\varphi}= & 4 a_{2} \sigma\left(2 a_{0}+4 \epsilon \sigma+\epsilon+5\right)-4 \sigma\left[6 a_{0} a_{2} b_{2} \sigma\right. \\
& -6 a_{0} a_{2} \sigma+a_{0} a_{2}-4 a_{0} b_{2} \sigma+2 a_{1} b_{2} \sigma \\
& +2 a_{2} \epsilon \sigma-8 a_{2} \sigma-3 b_{0}^{2} b_{2} \sigma-3 b_{0}^{2} \sigma \\
& \left.-6 b_{0} b_{2} \sigma-6 b_{0} \sigma+2 b_{2} \epsilon \sigma\right]\left(1+b_{2}\right)^{-1}
\end{aligned}
$$

The next step is to calculate the orbital period through direct integration of Eqs. (54) and (55) at the various orders of the metric expansion $[29,65]$. In particular, it is possible to distinguish the anomalistic period, which is the time for the 
particle to travel from one periastron to the next, and the sidereal period, which refers to the lapse in time between two successive passages across a line through the origin, fixed in space and lying in the orbital plane. For the sake of brevity, only the expression for the zeroth order case is given. The corresponding higher order expressions are straightforward but algebraically cumbersome to write explicitly. Upon using Eqs. (65) and (66), the proper time of the test particle is obtained as

$$
\begin{aligned}
{ }^{(0)} \tau= & \frac{p^{\frac{3}{2}} \sqrt{2-\left(e^{2}+3\right) \sigma}}{\sqrt{r_{0}}} \int_{\chi}^{2 \pi} d \chi^{\prime}\left(\frac{}{(0)}\left(\frac{d \varphi}{d \chi^{\prime}}\right)\right. \\
& \times \frac{1}{\left(e \cos \chi^{\prime}+1\right)^{2}\left[1-\sigma\left(e \cos \chi^{\prime}+1\right)\right]},
\end{aligned}
$$

and the observer time may then be expressed in terms of the proper time as

$$
{ }^{(0)} t=\sqrt{2}\left(\frac{\sqrt{(\sigma-1)^{2}-\sigma^{2} e^{2}}}{\sqrt{2-\left(e^{2}+3\right) \sigma}}\right){ }^{(0)} \tau \text {. }
$$

These expressions result in an elliptic integral which must be solved numerically. Expressing $t$ and $\tau$ in units of the Newtonian period

$$
P_{\mathrm{N}}=\left(\frac{8 \pi^{2} a^{3}}{G r_{0}}\right)^{\frac{1}{2}}
$$

of a Keplerian orbit, and the term multiplying the integral in Eq. (71) is given by

$$
\frac{P_{\mathrm{N}}}{2 \pi}\left(1-e^{2}\right)^{\frac{3}{2}} \frac{\sqrt{2-\left(e^{2}+3\right) \sigma}}{\sqrt{2}} .
$$

The integrals of Eqs. (71) and (72) give $t$ and $\tau$ in units of seconds upon restoring the proper physical unit values for $c$ and $G$. To calculate the above integrals, numerical quadrature must be used since for a very eccentric orbit, the integrand in (71) can be complex-valued. This can be avoided by reexpressing the integrals in terms of the eccentric anomaly $\psi$, which is here related to $\chi$ as

$$
(1+e \cos \chi)(1-e \cos \psi)=1-e^{2} .
$$

This definition is chosen in analogy with the classical case $[70,71]$. It is clear from the above calculations that one may obtain expressions for the orbital period in terms of the RZ parametrization at any and all orders by determining only the correct expression for $d \varphi / d \chi$.

\section{APPLICATION TO ASTROPHYSICAL TEST CASES}

In the following sections, both the RZ parametrization and the expressions derived so far for the periastron advance are tested. To do this, two sets of four test objects are considered. The first set is represented by four wellknown $S$-stars which have now been observed orbiting Sgr $A^{*}$ for more than a decade $[1,2,72-75]$. These first objects are Keplerian but serve the purpose of providing representative examples of how the parametrization can be employed. The second set of test objects is represented by four pulsar toy models. Their properties have been chosen to have a range of semimajor axes, reasonably high eccentricities, and moderate-to-short orbital periods. In this respect, these toy models are idealized but not altogether unrealistic: future advances in instrumental sensitivity could, in principle, enable the detection of Galactic center pulsars with such properties [13].

In modelling the toy pulsar-SMBH system, it is hereafter assumed that the mass of the central SMBH is known to some degree of precision. Whilst this is not the way pulsar timing normally works, since in such observations the mass of the $\mathrm{BH}$ is actually determined from the observations of the binary system, complementary observational data (e.g., multidecadal observations of $S$-stars [76]) can provide an independent measurement of the $\mathrm{BH}$ mass.

\section{A. Determining the periastron advance and orbital period}

In the case of $S$-stars, the accuracy at which the advance of the periastron can be measured places a lower limit on the eccentricity of orbits that are in the range $0.35<e<0.93$. For the purposes of this study, four specific $S$-stars are considered, namely, $S 1, S 2, S 9$, and $S 13$, which represent a broad range in both eccentricity and semimajor axis length [1,2,72-75].

The properties of these stars are collected in Table I, which also reports, besides the eccentricities $e$ and semimajor axes $a$, the values of the periastron advances $\Delta \varphi_{\mathrm{GR}}$ and ${ }^{(n)} \Delta \varphi$ at the various orders, $n$, in the RZ expansion. Note that in evaluating the periastron advance, specific values for the coefficients $\epsilon, a_{n}$, and $b_{n}$ must be specified since these coefficients cannot yet be constrained by astrophysical observations and hereafter chosen to be $a_{0}=2 a_{1}=4 a_{2}=\epsilon=10^{-3}$. The dependence of the periastron advance on $b_{n}$ is found to be extremely small. In particular, even in the case of the most optimistic model, Toy IV, the maximum relative difference in the periastron advance at second order is found to be less than one part in $10^{5}$. The coefficients $b_{n}$ therefore have a negligible effect on the pulsar dynamics, and they are hereafter taken to be zero.

Also reported in Table I are the values relative to the toy pulsar models, where models I, II, and III are in principle 
TABLE I. Values of the periastron advance $\Delta \varphi$ for different objects. Here, the numerical values for GR displacement, $\Delta \varphi_{\mathrm{GR}}$, and for the RZ parametrization (68)-(70) are shown. The table reports the measured values of the eccentricity $e$, semimajor axis $a$, and $\sigma=r_{\mathrm{g}} / \ell$, assuming that the gravitational radius is $r_{\mathrm{g}} \sim 6.64657 \times 10^{9} \mathrm{~m}=0.044$ AU for Sgr A* and that the values of coefficients are $a_{0}=2 a_{1}=4 a_{2}=\epsilon=10^{-3}$. Numbers in square brackets denote multiplicative powers of ten.

\begin{tabular}{|c|c|c|c|c|c|c|c|}
\hline Object & $e$ & $a[\mathrm{AU}]$ & $\sigma$ & $\Delta \varphi_{\mathrm{GR}}$ & ${ }^{(0)} \Delta \varphi$ & ${ }^{(1)} \Delta \varphi$ & ${ }^{(2)} \Delta \varphi$ \\
\hline$S 1$ & 0.358 & $3.29951[+3]$ & $1.55089[-5]$ & $2.91131[-4]$ & $2.92004[-4]$ & $2.92005[-4]$ & $2.92199[-4]$ \\
\hline$S 2$ & 0.876 & $9.79960[+2]$ & $2.01195[-4]$ & $3.77681[-3]$ & $3.78813[-3]$ & $3.78814[-3]$ & $3.79065[-3]$ \\
\hline$S 9$ & 0.825 & $2.33559[+3]$ & $6.01199[-5]$ & $1.12273[-3]$ & $1.12610[-3]$ & $1.12620[-3]$ & $1.12909[-3]$ \\
\hline$S 13$ & 0.395 & $9.53220[+2]$ & $5.54552[-5]$ & $1.04410[-3]$ & $1.04412[-3]$ & $1.04413[-3]$ & $1.04481[-3]$ \\
\hline Toy I & 0.800 & $1.75400[+2]$ & $7.03608[-4]$ & $1.32627[-2]$ & $1.33025[-2]$ & $1.33026[-2]$ & $1.33114[-2]$ \\
\hline Toy II & 0.800 & $4.38500[+1]$ & $2.81443[-3]$ & $5.30508[-2]$ & $5.32097[-2]$ & $5.32102[-2]$ & $5.32456[-2]$ \\
\hline Toy III & 0.786 & $5.00000[+0]$ & $2.32488[-2]$ & $4.38229[-1]$ & $4.39524[-1]$ & $4.39556[-1]$ & $4.39850[-1]$ \\
\hline Toy IV & 0.888 & $1.00000[+0]$ & $2.10110[-1]$ & $3.96047[+0]$ & $3.97069[+0]$ & $3.97417[+0]$ & $3.97695[+0]$ \\
\hline
\end{tabular}

already measurable with present radio-astronomical observations (see [77]), while Toy IV is, at the present time, an optimistic model. Since the RZ parametrization is constructed to be most accurate at the event horizon and at spatial infinity, the results presented in Table I are, albeit weakly, dependent on the specific form of the parametrization. However, given its inherent rapid convergence properties, by second order the RZ parametrization everywhere represents the chosen metric theory of gravity to an accuracy of better than 1\% [25]. In particular, looking at Table I one can establish how well the RZ parametrization works in the vicinity of the event horizon (this is especially true for Toy IV). In the case of the $S$-stars, on the other hand, the reported deviations from GR are all rather minute, and this is to be expected since their motions are essentially Keplerian.

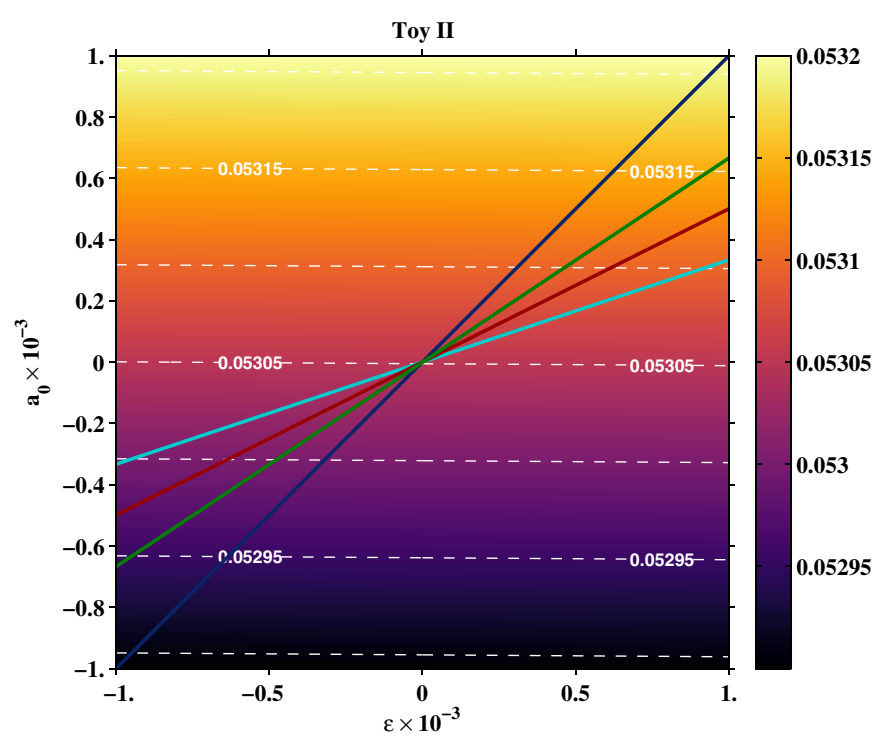

Figure 1 presents the results in Table I, highlighting how it is possible to use the results of the parametrization to distinguish between different theories of gravity. In particular, the color code in Fig. 1 indicates the values of the periastron advance at zeroth order, ${ }^{(0)} \Delta \varphi$, as a function of the only two free parameters in the lowest-order expansion of the parametrization, i.e., $a_{0}$ and $\epsilon$. The left and right panels in Fig. 1 refer to toy models II and IV, respectively.

In principle, from the observation of the periastron advance of a given star or pulsar, the value of $\Delta \varphi$ can be determined. If the observation is performed over much longer time scales, e.g., several decades as in the case of $S$ stars, the accuracy of this measurement is significantly improved. The eccentricity and semimajor axis of the orbit are then determined, and a contour plot akin to Fig. 1 is made for the given object. The observationally determined

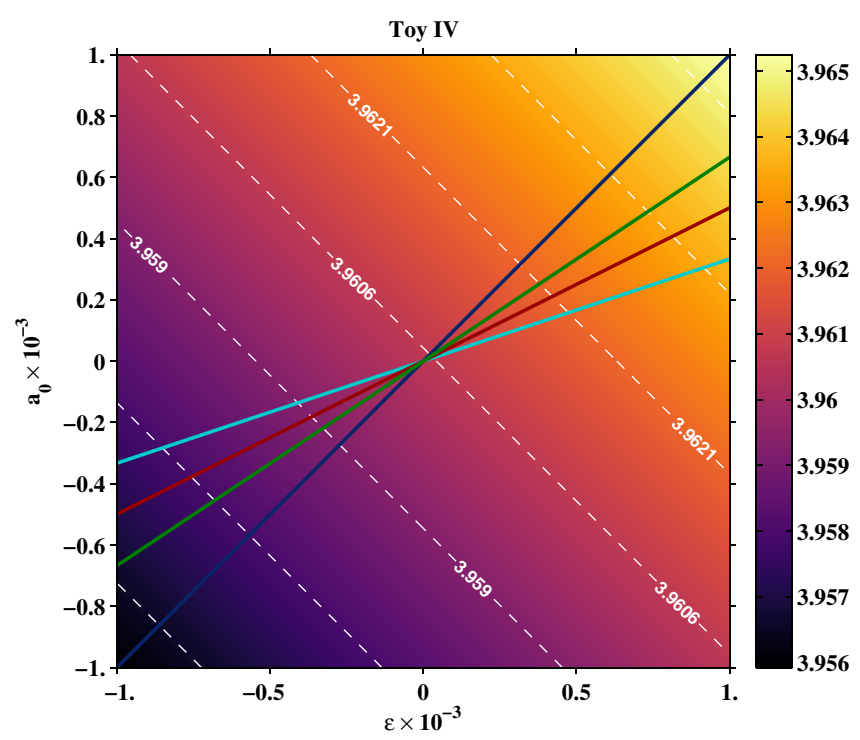

FIG. 1. Contour plot of periastron advance for the toy models II and IV with contour lines indicating the value of the periastron advance for a given value of $a_{0}$ and $\epsilon$. Overlapping curves indicate the four different theories, which are rewritten in terms of the RZ parametrization, i.e., (45) (blue line), (13) (green line), (32) (red line), and (21) (cyan line). Left panel: contour plot for toy model II. The separation between adjacent contours is $10^{-4}$. Right panel: contour plot for toy model IV. The separation between adjacent contours is $\sim 2.6 \times 10^{-3}$. 
TABLE II. Values of the orbital period and its relative difference for different objects. The Newtonian period $\mathrm{P}_{\text {Newton }}$, the GR orbital period $\mathrm{t}_{\mathrm{GR}}$ and the parameterised orbital period ${ }^{(0)} t,{ }^{(1)} t$ and ${ }^{(2)} t$ are given, assuming that $a_{0}=2 a_{1}=4 a_{2}=\epsilon=10^{-3}$. Numbers in square brackets denote multiplicative powers of ten.

\begin{tabular}{|c|c|c|c|c|c|}
\hline Object & $\mathrm{P}_{\text {Newton }}[\mathrm{s}]$ & $\mathrm{t}_{\mathrm{GR}}[\mathrm{s}]$ & ${ }^{(0)} t[\mathrm{~s}]$ & ${ }^{(1)} t[\mathrm{~s}]$ & ${ }^{(2)} t[\mathrm{~s}]$ \\
\hline$S 1$ & $2.81918[+9]$ & $2.81924[+9]$ & $2.82170[+9]$ & $2.82171[+9]$ & $2.82202[+9]$ \\
\hline$S 2$ & $4.37764[+8]$ & $4.37794[+8]$ & $4.37896[+8]$ & $4.37896[+8]$ & $4.38220[+8]$ \\
\hline$S 9$ & $1.67898[+9]$ & $1.67903[+9]$ & $1.67956[+9]$ & $1.67958[+9]$ & $1.68065[+9]$ \\
\hline$S 13$ & $4.37764[+8]$ & $4.37794[+8]$ & $4.38164[+8]$ & $4.38166[+8]$ & $4.38225[+8]$ \\
\hline Toy I & $3.45546[+7]$ & $3.45678[+7]$ & $3.45802[+7]$ & $3.45805[+7]$ & $3.46012[+7]$ \\
\hline Toy II & $4.31933[+6]$ & $4.32593[+6]$ & $4.32749[+6]$ & $4.32750[+6]$ & $4.33012[+6]$ \\
\hline Toy III & $1.66308[+5]$ & $1.68621[+5]$ & $1.68687[+5]$ & $1.68688[+5]$ & $1.68789[+5]$ \\
\hline Toy IV & $1.48750[+4]$ & $1.65483[+4]$ & $1.65521[+4]$ & $1.65539[+4]$ & $1.65751[+4]$ \\
\hline
\end{tabular}

value of $\Delta \varphi$ is then represented as a contour line in this plot, and deviations from GR (located at the point $\left(a_{0}, \epsilon\right)=$ $(0,0)$ in all plots) are manifest. Different colored lines indicate the constraints placed on $a_{0}$ and $\epsilon$ by the different theories of gravity considered here, namely, Eq. (45) (blue), Eq. (13) (green), Eq. (32) (red), and Eq. (21) (cyan). Such lines may be constructed for any desired theory of gravity. In principle, the intersection of the object's contour line with any and all constructed (herein coloured) theory lines can provide an estimate of both the deviation from GR and which potential theories are more strongly (or weakly) constrained.

Considerations similar to the ones made so far for the periastron advance can also be made for the observed orbital period. In particular, Table II and Fig. 2 provide information analogous to that presented in Table I and in Fig. 1 but now for the orbital period. More specifically, Table II reports the orbital periods at different orders in the expansion (see Sec. VIA). As expected, in the case of $S$-stars the deviations from GR are very small since their motion is effectively Keplerian, while larger deviations are seen for the pulsar toy models and, in particular, for model IV. Table II also demonstrates that the orbital period appears to be a more sensitive parameter than the periastron advance (i.e., deviations from GR are much more pronounced).

Figure 2 presents a contour plot of the values of the orbital period at the zeroth order in the parametrization, ${ }^{(0)} t$, as a function of the coefficients $a_{0}$ and $\epsilon$. Analogous to Fig. 1, different colored lines represent the corresponding values for $a_{0}$ and $\epsilon$ predicted at this order by the aforementioned different theories.

As seen in Figs. 1 and 2, the allowed range of $a_{0}$ and $\epsilon$ is not yet bounded, and thus (although increasingly unlikely for larger values of $a_{0}$ and $\epsilon$ ), it is not possible to strongly constrain a particular theory. However, simultaneous
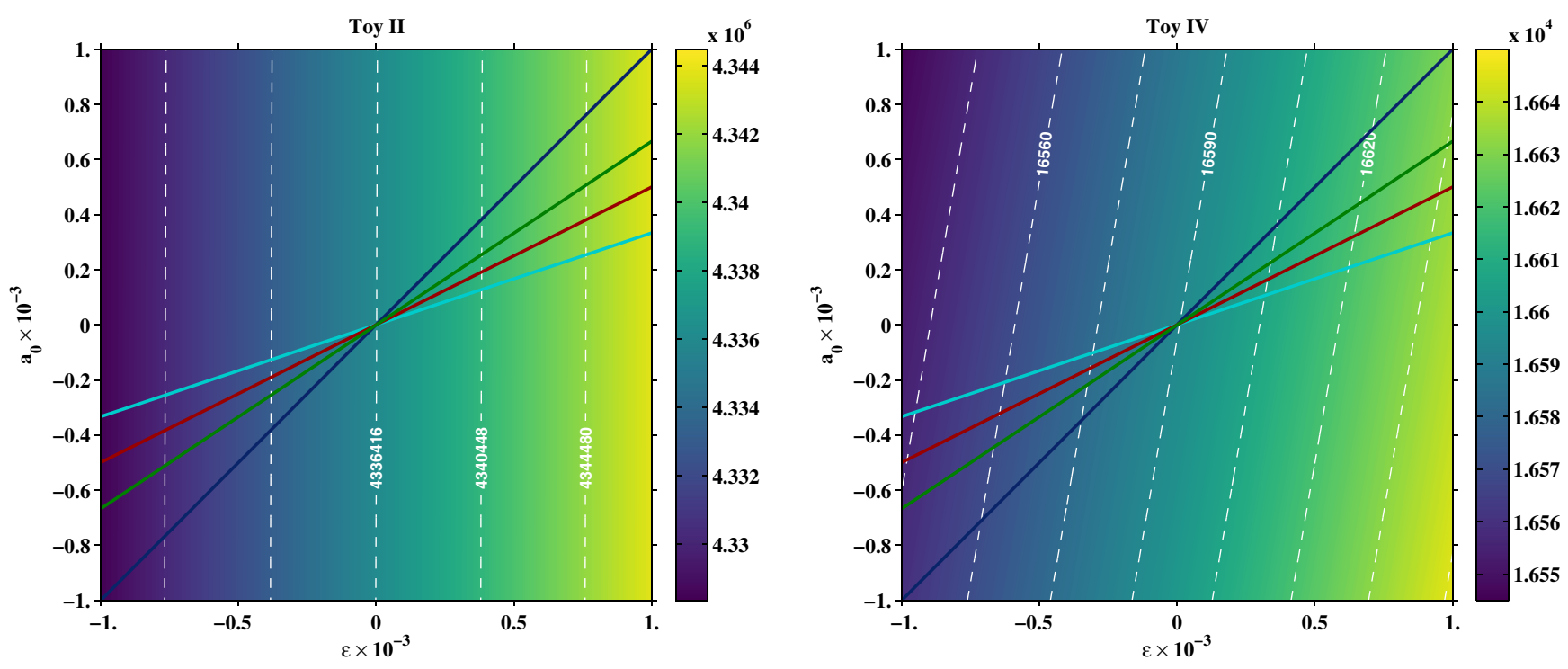

FIG. 2. Contour plot of the orbital period and curves as defined in Fig. 1. Left panel: contour for the toy model II. The separation between adjacent contours is $4032 \mathrm{~s}$. Right panel: contour for the toy model IV. The separation between adjacent contours is $15 \mathrm{~s}$. 

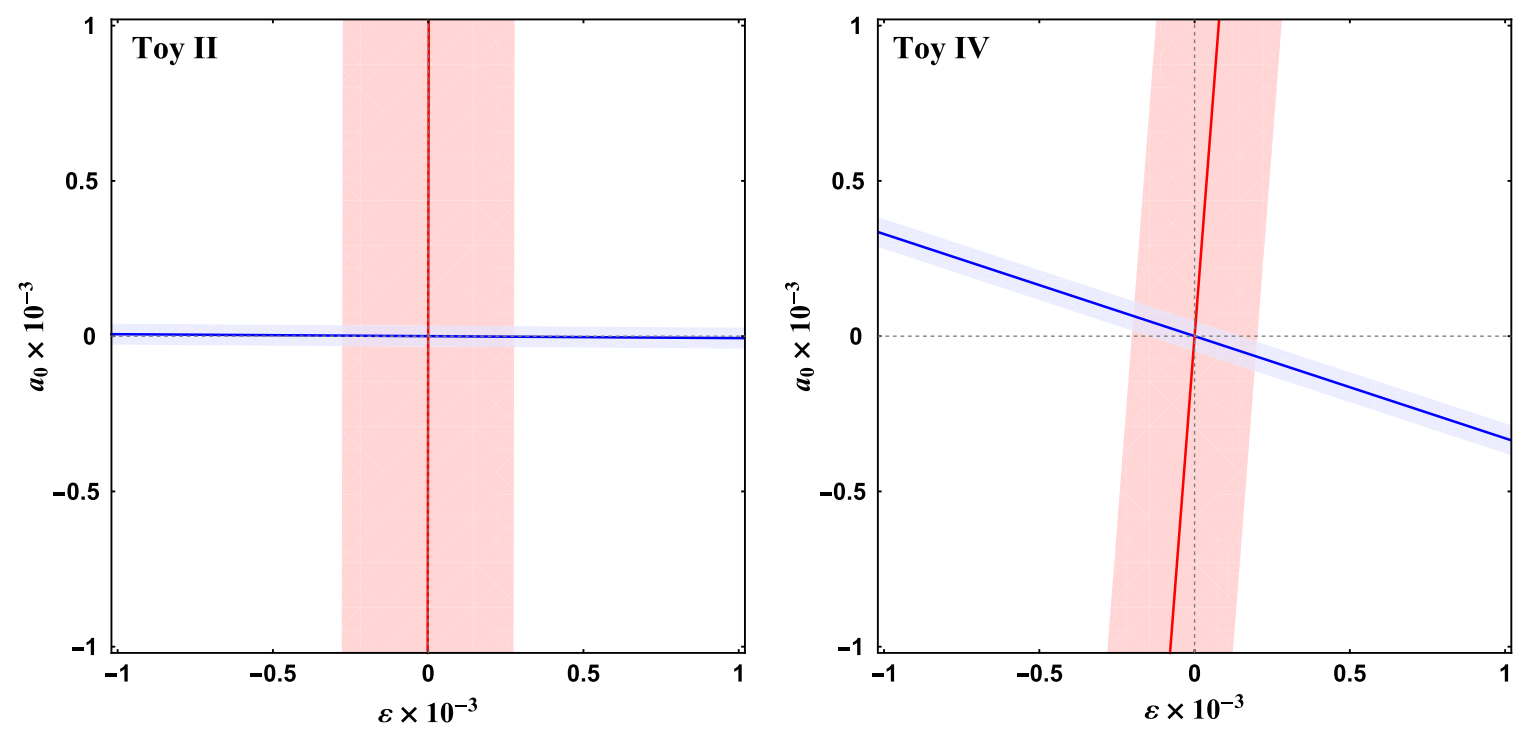

FIG. 3. Constraints set by the pulsar orbits for toy models II and IV in the EMDA metric (39) at zeroth order, with the dilaton parameter fixed at $\hat{b}=5 \times 10^{-8}$. The values of the coefficients $a_{0}$ and $\epsilon$ are constrained by the observations of the periastron advance (light-blue shaded areas around the solid blue line) and of the orbital period (light-red shaded areas around the solid red line) assuming an accuracy of $10^{-4}$.

observation of both the periastron advance and orbital period of the object can (for a given accuracy) place constraints on the allowed range of $a_{0}$ and $\epsilon$. This in turn enables, upon reexamining Figs. 1 and 2, not only much more stringent constraints to be imposed on given theories but also in principle the possibility to rule out certain theories entirely. This is illustrated in Fig. 3 for the EMDA BH (assuming $\hat{b}=5 \times 10^{-8}$ ) using models Toy II and Toy IV, where the periastron advance (light-blue shaded areas around the solid blue line) and of the orbital period (lightred shaded areas around the red line) assuming an accuracy of $10^{-4}$. The specific case of an EMDA BH was chosen in this example since it is not merely an extension of GR.

In summary, the results reported in Figs. 1-3 demonstrate that by using a general description of test-particle motion in arbitrary BH spacetimes, such as the RZ parameterization, future observations of pulsars near a SMBH can help impose tighter constraints on different theories of gravity, and even potentially facilitate ruling out certain theories (and, by extension, related classes and extensions thereof) entirely.

\section{B. Constraining EMDA and $\boldsymbol{f}(\boldsymbol{R})$ theories}

In this subsection, an illustrative example demonstrating the facility of the parametric framework to constrain the parameters of two different theories of gravity, namely, EMDA and $f(R)$, is presented. These two theories have been chosen since they are not only distinctly different both mathematically and physically but also provide a strong contrast in the ability of the RZ parametrization to constrain theory-dependent parameters.
Figures 4 and 5 present the deviations from GR of the periastron advance as a function of the semimajor axis length, for models Toy I and Toy II (Fig. 4) and model Toy IV (Fig. 5). The left panels in both figures correspond to $f(R)$ and the right panels to EMDA. Multiple colored curves, colored from violet through to red, denote, for a fixed value of the theory parameter, how the relative difference in the periastron advance varies as a function of the semimajor axis length. These 51 colored lines are uniformly logarithmically spaced between the stated parameter value in the upper right of each panel (uppermost red line) and 0.01 times that value (bottom-most violet line), i.e., 25 lines per decade in the theory parameter.

In Fig. 4, the relative differences in the periastron advance for models Toy I and Toy II are shown for $f(R)$ (left panel) and EMDA (right panel). The two vertical lines in both panels denote the semimajor axis position of models Toy I (blue) and Toy II (magenta). The horizontal black dashed line at $10^{-7}$ represents a potential astrophysical measurement precision [77]. It is immediately clear that for models Toy I and Toy II the relative differences in the periastron advance are insensitive to the semimajor axis length and practically indistinguishable, as is evident from the horizontal, parallel theory parameter lines. This can be interpreted as near-Keplerian pulsar motion, i.e., the weakfield limit of the RZ parametrization. The intersection of the uppermost plotted theory parameter line with the vertical blue and magenta lines provides an upper limit for the theory parameters. For the $f(R)$ case, this yields $\alpha<1.288 \times 10^{-4}$, whereas for the EMDA case this yields the much smaller value of $\hat{b}<6.68 \times 10^{-8}$, nearly four orders of magnitude smaller than that in the $f(R)$ case. 

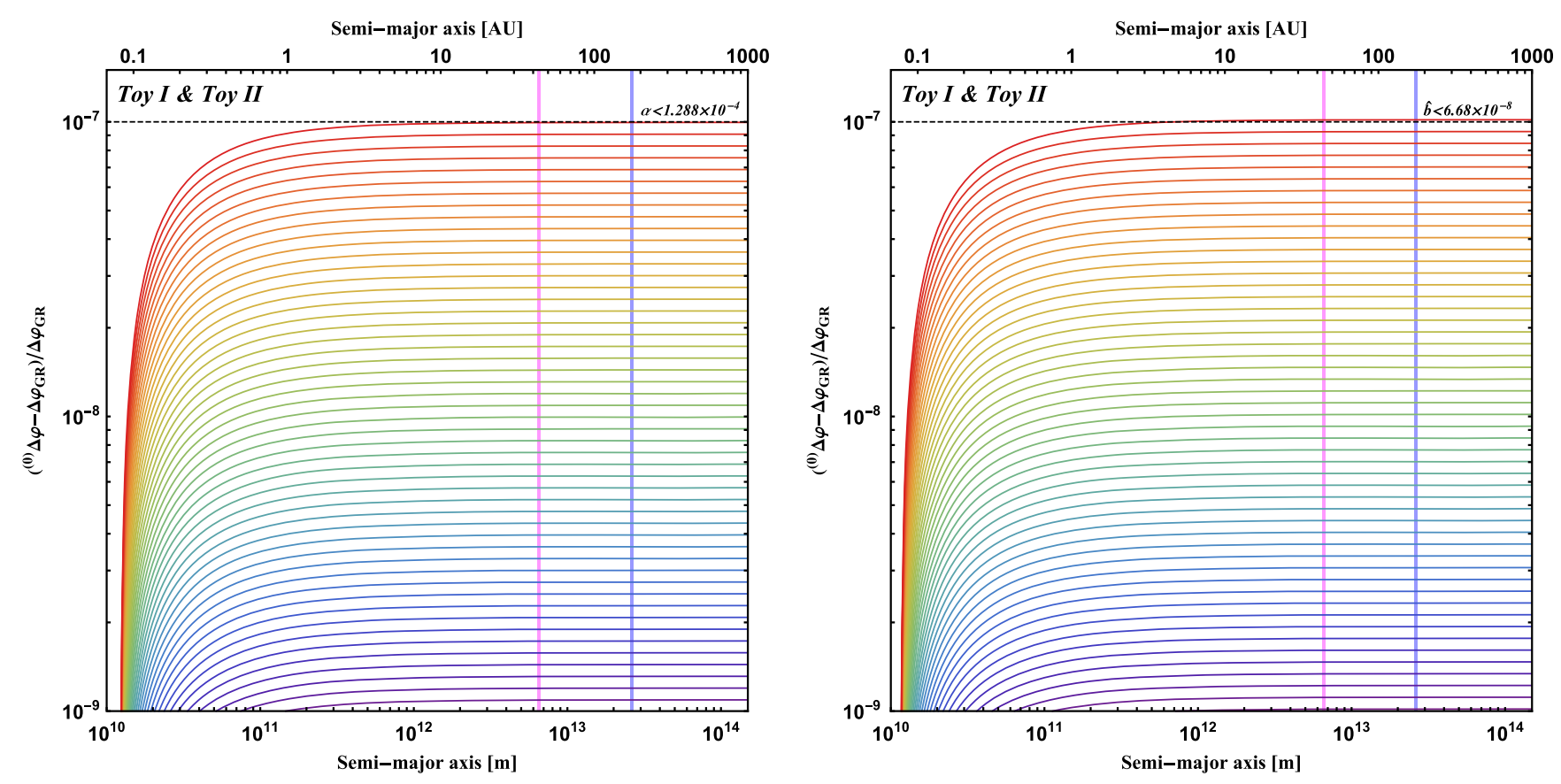

FIG. 4. Relative difference of the zeroth order periastron advance with respect to the GR value plotted as a function of semimajor axis distance for toy models I and II in the case of $f(R)$ (left panel) and EMDA (right panel). Different colored lines indicate, for a fixed value of the theory parameter $(\alpha$ or $\hat{b}$ ), the variation in this relative difference as a function of the semimajor axis. The 51 lines are equally spaced (logarithmically) between the stated inset upper limits of $\alpha \leq 1.288 \times 10^{-4}$ and $\hat{b} \leq 6.68 \times 10^{-8}$, and 0.01 times those values, i.e., 25 equally spaced values per decade. The vertical solid lines at 175.4 AU (blue) and 43.85 AU (magenta) indicate the semimajor axis positions of toy models I and II, respectively.
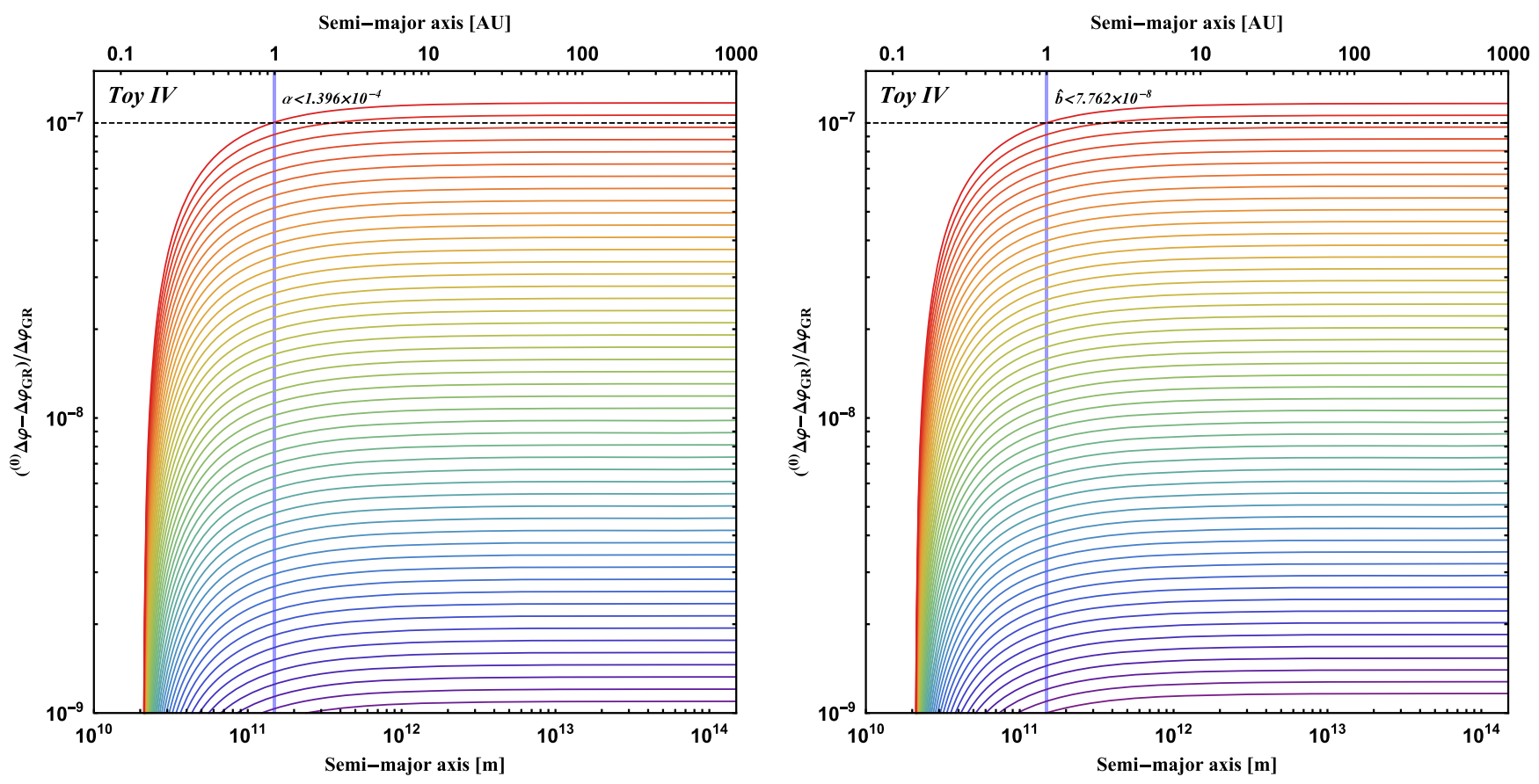

FIG. 5. Relative difference of the zeroth order periastron advance with respect to the GR value plotted as a function of semi-major axis distance for toy models IV in the case of $f(R)$ (left panel) and EMDA (right panel). Different colored lines are as discussed in Fig. 4, with upper limits of $\alpha \leq 1.396 \times 10^{-4}$ and $\hat{b} \leq 7.762 \times 10^{-8}$. The vertical solid line (blue) at 1 AU denotes the semimajor axis position of toy model IV. 
In Fig. 5, the same analysis in Fig. 4 is repeated for model Toy IV. In this case, the vertical blue line denotes the semimajor axis position of model Toy IV. Since this model places the pulsar much closer to the event horizon of the $\mathrm{BH}(1 \mathrm{AU})$, and as is clear from the theory parameter lines steeper gradients, the pulsar motion can be considered as occurring in the transition region between the weak-field and strong-field regimes. For the $f(R)$ case, this yields $\alpha<1.396 \times 10^{-4}$, whereas for the EMDA case this yields $\hat{b}<7.762 \times 10^{-8}$.

In particular, one can see from Figs. 4 and 5 that it is possible to provide constraints on the two theories. More specifically, one can place an upper limit on the value of the parameters $\alpha$ and $\hat{b}$. It is assumed that the error on the measurements is of the order of $10^{-7}$ [77]. Figures 4 and 5 present the relative differences from ${ }^{(0)} \Delta \varphi$ at first order of the expansion in GR with respect to the semimajor axes for the four toy models for both theories. What emerges from the figures is that the deviations from GR are much more pronounced if one considers, as seen before, small semimajor axes and high eccentricities. In addition, one may also estimate the various values of the parameters that characterize the specific theory through the location of the different level curves.

It can be seen from Figs. 4 and 5 that for a given measurement precision, as the pulsar semimajor axis location is shifted towards the $\mathrm{BH}$ event horizon, the range over which the theory parameter can be probed (and effectively constrained) is increased. Therefore, a pulsar orbiting in the immediate vicinity of a $\mathrm{BH}$ event horizon (i.e., in the truly strong-field regime) can provide much tighter constraints on the theory parameters.

From Figs. 4 and 5, it follows that $f(R)$ gravity can be more stringently constrained using pulsars than EMDA theory. This result is reassuring given that $f(R)$ is an extension of GR, rather than a truly distinct theory of gravity like EMDA theory. If a pulsar near Sgr A*'s event horizon is detected, such observations will aid in fixing ranges of validity and values of the parameters of any theory under consideration. It is clear that in order to be proven useful, such a pulsar must be close to the SMBH, with an orbital period of only a few hours or less. Observational detection of such pulsars is in principle achievable with present day radio telescopes.

\section{CONCLUSIONS}

Although GR has proven to be a very reliable theory of gravity in several different fields of application and across several different scales, it is not the only one. Indeed, there exist other theories of gravity that reproduce the prediction of GR. There is therefore a need, both theoretically and observationally, to impose constraints on such new theories of gravity and possibly to exclude some of them.
Owing to their very narrow mass range, extreme compactness, and rapid, stable rotation periods, pulsars are one of the best candidates to probe strong-field gravity in the truly nonlinear regime. Pulsar-timing measurements, with their inherent high precision, have already proven to be highly sensitive and accurate in the weak-field regime [78]. The detection of a pulsar in the strong-field regime, i.e., near a $\mathrm{BH}$ event horizon, would enable not just a highly accurate determination of the $\mathrm{BH}$ properties but also provide an accurate probe of the spacetime structure and geometry.

Given the rapid increase in recent efforts to perform astronomical observations of the Galactic center, the prospect of detecting a pulsar orbiting in close proximity to $\mathrm{Sgr} \mathrm{A}^{*}$ is promising. Such a detection would provide the most accurate measurements of the physical parameters (e.g., mass, spin, and even quadrupole moment) of Sgr A* [79]. Theoretical studies of pulsar motion and timing in both GR and alternative theories of gravity are therefore of great importance. However, given the breadth of available theories of gravity in the present literature, it is most expedient to perform any such studies in a manner which assumes neither a specific theory of gravity nor any particular solution to any particular theory. It is also most desirable to have a representation in which the classical GR limit is recovered.

Consequently, this study has presented an analysis of test-particle (i.e., pulsar) dynamics in several different $\mathrm{BH}$ spacetimes, using a theory-independent approach. This approach makes use of a general mathematical representation of BH spacetimes. In the case of spherically symmetric spacetimes, as those considered in the present study, a rapidly convergent continued fraction expansion in terms of a compactified radial coordinate has been employed.

Using this parametrization, general expressions for the dynamics of a test particle in general $\mathrm{BH}$ spacetimes were derived. In particular, general algebraic expressions for the advance of the periastron and the orbital period at different orders of the parametrization have been presented. This formalism was applied to two sets of astrophysical test cases: (i) four particular $S$-stars, which have been observed orbiting Sgr A* in the infrared, and (ii) four hypothetical toy models for pulsars.

It was shown that in the case of $S$-stars, deviations from GR are negligible, and therefore, it is not possible to use such objects to test the underlying theory of gravity. The classical GR results of $S$-star periastron advance and orbital period observations are well-reproduced within the paramterization, and it was shown that deviations from GR are negligible in this case. Therefore $S$-stars, while useful in providing constraints on the mass and distance of the central $\mathrm{SMBH}$, are of limited use in probing strong-field gravity.

Next, the periastron advance and orbital period properties of four pulsar toy models were investigated. It was shown that, in contrast to $S$-stars, pulsars with smaller 
semimajor axis lengths (i.e., orbiting closer to Sgr $\mathrm{A}^{*}$ ) indeed exhibit quantifiable deviations from GR. It was demonstrated that separate measurements of the periastron advance and of the orbital period of the pulsar enable two independent means through which to determine the parameters $a_{0}$ and $\epsilon$ that describe, at lowest order, a generic $\mathrm{BH}$ spacetime in the RZ parametrization. Hence, such measurements offer the possibility to constrain any particular theory of gravity.

In order to attempt to rule out certain theories, an example of the simultaneous calculation of the periastron advance and orbital period (complete with accuracy errors) was presented. This defines an inequality through which a region of overlap in the shared parameter space of both observational quantities can be determined, imposing tighter constraints on $a_{0}$ and $\epsilon$. Such constraints can then be overlaid demonstrating that, in principle, given sufficient observational sensitivity, certain theories of gravity (and even extensions or classes thereof) can be immediately ruled out.

In particular, the cases of $f(R)$ and EMDA theories were employed to show that measurements of the relative difference (from GR) of the periastron advance can provide another avenue through which to constrain the parameters of different theories of gravity. Moreover, it was found that
$f(R)$ theories are much more stringently constrained than EMDA theory. Given that the $f(R)$ theory considered in this study is, at its core, an extension of GR, this result, while not necessarily obvious, stands to reason given that this particular extension of GR includes higher-order curvature invariants. Therefore, using pulsar observations presents the possibility to strongly constrain the parameters of all theories which are purely geometrical (i.e., not containing exotic particles and scalar fields).

\section{ACKNOWLEDGMENTS}

We thank Norbert Wex for numerous helpful discussions, comments, and for helping construct the pulsar toy models. We also thank C. Fromm, H. Olivares and A. Zhindenko for their support. Support comes from the ERC Synergy Grant "BlackHoleCam-Imaging the Event Horizon of Black Holes" (Grant No. 610058), "NewCompStar", COST Action MP1304, the LOEWEProgram in the Helmholtz International Center (HIC) for FAIR, and the European Union's Horizon 2020 Research and Innovation Programme (Grant No. 671698) (call FETHPC-1-2014, project ExaHyPE). Z. Y. acknowledges support from an Alexander von Humboldt Fellowship.
[1] A. Eckart and R. Genzel, Nature (London) 383, 415 (1996).

[2] A. M. Ghez, S. Salim, N. N. Weinberg, J. R. Lu, T. Do, J. K. Dunn, K. Matthews, M. R. Morris, S. Yelda, E. E. Becklin, T. Kremenek, M. Milosavljevic, and J. Naiman, Astrophys. J. 689, 1044 (2008).

[3] S. Capozziello and M. de Laurentis, Phys. Rep. 509, 167 (2011).

[4] C. T. Cunningham and J. M. Bardeen, Astrophys. J. 183, 237 (1973).

[5] H. Falcke, F. Melia, and E. Agol, Astrophys. J. Lett. 528, L13 (2000).

[6] A. Grenzebach, V. Perlick, and C. Lämmerzahl, Phys. Rev. D 89, 124004 (2014).

[7] A. A. Abdujabbarov, L. Rezzolla, and B. J. Ahmedov, Mon. Not. R. Astron. Soc. 454, 2423 (2015).

[8] Z. Younsi, A. Zhidenko, L. Rezzolla, R. Konoplya, and Y. Mizuno, Phys. Rev. D 94, 084025 (2016).

[9] S. S. Doeleman, J. Weintroub, A. E. E. Rogers, R. Plambeck, R. Freund, R. P. J. Tilanus, P. Friberg, L. M. Ziurys, J. M. Moran, B. Corey, K. H. Young, D. L. Smythe, M. Titus, D. P. Marrone, R. J. Cappallo et al., Nature (London) 455, 78 (2008).

[10] K. Akiyama, R.-S. Lu, V. L. Fish, S. S. Doeleman, A. E. Broderick, J. Dexter, K. Hada, M. Kino, H. Nagai, M. Honma, M. D. Johnson, J. C. Algaba, K. Asada, C. Brinkerink, R. Blundell et al., Astrophys. J. 807, 150 (2015).
[11] V. L. Fish, M. D. Johnson, S. S. Doeleman, A. E. Broderick, D. Psaltis, R.-S. Lu, K. Akiyama, W. Alef, J. C. Algaba, K. Asada, C. Beaudoin, A. Bertarini, L. Blackburn, R. Blundell, G. C. Bower et al., Astrophys. J. 820, 90 (2016).

[12] C. Goddi, H. Falcke, M. Kramer, L. Rezzolla, C. Brinkerink, T. Bronzwaer, J. R. J. Davelaar, R. Deane, M. de Laurentis, G. Desvignes, R. P. Eatough, F. Eisenhauer, R. Fraga-Encinas, C. M. Fromm, S. Gillessen et al., Int. J. Mod. Phys. D 26, 1730001 (2017).

[13] D. R. Lorimer and M. Kramer, Handbook of Pulsar Astronomy, (Cambridge University Press, Cambridge, 2012).

[14] P. C. C. Freire, N. Wex, G. Esposito-Farèse, J. P. W. Verbiest, M. Bailes, B. A. Jacoby, M. Kramer, I. H. Stairs, J. Antoniadis, and G. H. Janssen, Mon. Not. R. Astron. Soc. 423, 3328 (2012).

[15] L. Iorio, Universe 4, 59 (2018).

[16] L. Iorio, Mon. Not. R. Astron. Soc. 472, 2249 (2017).

[17] P. A. R. Ade, N. Aghanim, M. Arnaud, M. Ashdown, J. Aumont, C. Baccigalupi, A. J. Banday, R. B. Barreiro, J. G. Bartlett et al. (Planck Collaboration), Astron. Astrophys. 594, A13 (2016).

[18] P. A. R. Ade, R. W. Aikin, D. Barkats, S. J. Benton, C. A. Bischoff, J. J. Bock, J. A. Brevik, I. Buder, E. Bullock, C. D. Dowell, L. Duband, J. P. Filippini, S. Fliescher, S. R. Golwala, M. Halpern et al. (BICEP2 Collaboration), Phys. Rev. Lett. 112, 241101 (2014). 
[19] F. Zwicky, Helv. Phys. Acta 6, 110 (1933).

[20] S. Capozziello and M. De Laurentis, Ann. Phys. (Berlin) 524, 545 (2012).

[21] T. Johannsen and D. Psaltis, Phys. Rev. D 83, 124015 (2011).

[22] N. Lin, N. Tsukamoto, M. Ghasemi-Nodehi, and C. Bambi, Eur. Phys. J. C 75, 599 (2015).

[23] C. Bambi, Rev. Mod. Phys. 89, 025001 (2017).

[24] Y. N. Obukhov and D. Puetzfeld, Proceedings, 524th WEHeraeus-Seminar: Equations of Motion in Relativistic Gravity (EOM 2013): Bad Honnef, Germany, February 17-23, 2013 Fund. Theor. Phys. 179, 67 (2015).

[25] L. Rezzolla and A. Zhidenko, Phys. Rev. D 90, 084009 (2014).

[26] R. Konoplya, L. Rezzolla, and A. Zhidenko, Phys. Rev. D 93, 064015 (2016).

[27] Y. Mizuno et al. (to be published).

[28] K. D. Kokkotas, R. A. Konoplya, and A. Zhidenko, Phys. Rev. D 96, 064007 (2017).

[29] S. Chandrasekhar, The Mathematical Theory of Black Holes (Oxford University Press, Oxford, England, 1983).

[30] S. Nojiri and S. D. Odintsov, Eur. Phys. J. C 71, 1801 (2011).

[31] Y.-F. Cai, S. Capozziello, M. De Laurentis, and E. N. Saridakis, Rep. Prog. Phys. 79, 106901 (2016).

[32] T. P. Sotiriou and V. Faraoni, Rev. Mod. Phys. 82, 451 (2010).

[33] Z. Younsi and A. Grenzebach, in Proceedings, 14th Marcel Grossmann Meeting on Recent Developments in Theoretical and Experimental General Relativity, Astrophysics, and Relativistic Field Theories (MG14) (In 4 Volumes), Rome, Italy, July 12-18, 2015 (World Scientific, Singapore, 2017) Vol. 4, pp. 3525-3530.

[34] C. Brans and R. H. Dicke, Phys. Rev. 124, 925 (1961).

[35] T. Damour, in Proceedings of the 2nd Canadian Conference on General Relativity and Relativistic Astrophysics, edited by A. Coley, C. Dyer, and T. Tupper (1988) pp. 315-334.

[36] T. Damour and G. Esposito-Farèse, Phys. Rev. D 58, 042001 (1998).

[37] K. S. Thorne and J. J. Dykla, Astrophys. J. Lett. 166, L35 (1971).

[38] H. Kim, Phys. Rev. D 60, 024001 (1999).

[39] M. Campanelli and C. O. Lousto, Int. J. Mod. Phys. D 02, 451 (1993).

[40] L. Vanzo, S. Zerbini, and V. Faraoni, Phys. Rev. D 86, 084031 (2012).

[41] S. Capozziello and M. Francaviglia, Gen. Relativ. Gravit. 40, 357 (2008).

[42] C. M. Will, Theory and Experiment in Gravitational Physics (Cambridge University Press, Cambridge, UK, 1993) p. 396. ISBN 0521439736.

[43] A.S. Eddington, The Mathematical Theory of Relativity (Cambridge University Press, Cambridge, UK, 1923).

[44] S. Capozziello and A. Troisi, Phys. Rev. D 72, 044022 (2005).

[45] S. Capozziello, A. Stabile, and A. Troisi, Mod. Phys. Lett. A 21, 2291 (2006).

[46] S. Capozziello, M. de Laurentis, S. Nojiri, and S. D. Odintsov, Gen. Relativ. Gravit. 41, 2313 (2009).

[47] T. Damour, arXiv:0705.3109.
[48] M. De Laurentis and I. De Martino, Mon. Not. R. Astron. Soc. 431, 741 (2013).

[49] M. De Laurentis and S. Capozziello, Astropart. Phys. 35, 257 (2011).

[50] M. De Laurentis, R. De Rosa, F. Garufi, and L. Milano, Mon. Not. R. Astron. Soc. 424, 2371 (2012).

[51] M. De Laurentis, M. Paolella, and S. Capozziello, Phys. Rev. D 91, 083531 (2015).

[52] N. D. Birell and P. C. W. Davis, Quantum Fields in Curved Space (Cambridge University Press, Cambridge, 1982).

[53] D. Borka, S. Capozziello, P. Jovanović, and V. Borka Jovanović, Astropart. Phys. 79, 41 (2016).

[54] S. Capozziello, D. Borka, P. Jovanović, and V. B. Jovanović, Phys. Rev. D 90, 044052 (2014).

[55] A. Hees, A. M. Ghez, T. Do, J. R. Lu, M. R. Morris, E. E. Becklin, G. Witzel, A. Boehle, S. Chappell, Z. Chen, D. Chu, A. Ciurlo, A. Dehghanfar, E. Gallego-Cano, A. Gautam et al., arXiv:1705.10792.

[56] D. V. Gal'tsov and O. V. Kechkin, Phys. Rev. D 50, 7394 (1994).

[57] G. Clément and D. Gal'tsov, Phys. Rev. D 54, 6136 (1996).

[58] A. García, D. Galtsov, and O. Kechkin, Phys. Rev. Lett. 74, 1276 (1995).

[59] G. W. Gibbons and K.-I. Maeda, Nucl. Phys. B B298, 741 (1988).

[60] D. Garfinkle, G. T. Horowitz, and A. Strominger, Phys. Rev. D 43, 3140 (1991).

[61] G. T. Horowitz and A. Strominger, Nucl. Phys. B360, 197 (1991).

[62] A. Shapere, S. Trivedi, and F. Wilczek, Mod. Phys. Lett. A 06, 2677 (1991).

[63] A. Sen, Phys. Rev. Lett. 69, 1006 (1992).

[64] L. D. Landau and E. M. Lifshitz, Mechanics, (Pergamon Press, Oxford, 1976) Vol. 1.

[65] A. E. Roy, Orbital Motion, 4th ed. (Institute of Physics Publishing, Bristol, UK, 2005) ISBN 0-7503-1015-6, 2005, Vol. XVIII, p. 526.

[66] C. Darwin, Proc. R. Soc. London Sect. A 263, 39 (1961).

[67] P. A. Geisler, Astron. J. 68, 715 (1963).

[68] V. P. Frolov and I. D. Novikov, Black Hole Physics, Basic Concepts and New Developments (Kluwer Academic Publishers, Dordrecth, The Netherlands, 1998).

[69] M. Maggiore, Gravitational Waves: Volume 1: Theory and Experiments (Oxford University Press, New York, 2007).

[70] T. Damour and N. Deruelle, Ann. Inst. Henri Poincaré Phys. Théor. 43, 107 (1985).

[71] T. Damour and N. Deruelle, Ann. Inst. Henri Poincaré Phys. Théor. 44, 263 (1986).

[72] A. Eckart and R. Genzel, Mon. Not. R. Astron. Soc. 284, 576 (1997).

[73] S. Gillessen, F. Eisenhauer, S. Trippe, T. Alexander, R. Genzel, F. Martins, and T. Ott, Astrophys. J. 692, 1075 (2009).

[74] S. Gillessen, F. Eisenhauer, T. K. Fritz, H. Bartko, K. Dodds-Eden, O. Pfuhl, T. Ott, and R. Genzel, Astrophys. J. Lett. 707, L114 (2009).

[75] A. M. Ghez, B. L. Klein, M. Morris, and E. E. Becklin, Astrophys. J. 509, 678 (1998).

[76] A. Boehle, A. M. Ghez, R. Schödel, L. Meyer, S. Yelda, S. Albers, G. D. Martinez, E. E. Becklin, T. Do, J. R. Lu, 
K. Matthews, M. R. Morris, B. Sitarski, and G. Witzel, Astrophys. J. 830, 17 (2016).

[77] K. Liu, N. Wex, M. Kramer, J. M. Cordes, and T. J. W. Lazio, Astrophys. J. 747, 1 (2012).
[78] R. N. Manchester, Int. J. Mod. Phys. D 22, 1341007 (2013).

[79] D. Psaltis, N. Wex, and M. Kramer, Astrophys. J. 818, 121 (2016). 